\title{
Abnormal vulval lesions: diagnosis and management
}

\author{
Aarti Kothari $^{1 *}$, Usha Parekh ${ }^{1}$, Rashmi Mahajan $^{2}$
}

\author{
${ }^{1}$ Department of Obstetrics and Gynecology, Dhiraj General Hospital, Waghodia, Vadodara, Gujarat, India \\ ${ }^{2}$ Department of Dermatology, Dhiraj General Hospital, Waghodia, Vadodara, Gujarat, India
}

Received: 18 July 2016

Accepted: 17 August 2016

\author{
*Correspondence: \\ Dr. Aarti Kothari, \\ E-mail: aartikothari13@gmail.com
}

Copyright: (c) the author(s), publisher and licensee Medip Academy. This is an open-access article distributed under the terms of the Creative Commons Attribution Non-Commercial License, which permits unrestricted non-commercial use, distribution, and reproduction in any medium, provided the original work is properly cited.

\begin{abstract}
Vulval symptoms are commonly found and cause considerable distress for women. Symptoms are often chronic and can significantly affect the quality of women's lives including sexual function and wellbeing. Despite the frequency of vulval symptoms, women often find it difficult to seek medical advice due to multiple reasons, mostly cultural and emotional. This part is not easily self-observable neither are women aware of the fact that a vulvar lesion could also be a manifestation of a systemic disease. The idea behind this study was to identify whether presenting vulval lesion is dermatological or non-dermatological and to control the symptoms, to identify nature of the various vulvar lesions (inflammatory or neoplastic) and to identify those skin lesions not responding to treatment which require biopsy and further management. It was a clinical descriptive study. Cases included women with vulvar lesions who attended Gynaec OPD at Dhiraj General Hospital affiliated to S.B.K.S. Medical Institute and Research Centre of Vadodara. After taking their informed consent, all the women who had symptoms like vaginal discharge, itching or discomfort associated a with vulvar lesion, visible lesion or growth over vulva were thoroughly investigated to rule out any systemic illness causing vulvar lesion following which biopsy was taken if solid growth or a non-healing lesion was present. Treatment was done according to the lesions. In the presence of vulvar complains, a diagnostic pathway including systematic physical examination is always mandatory to detect SCC early. A non-healing lesion must be subjected to biopsy mandatorily. Women should be educated to avoid ODC drugs or creams when they face vulval lesions.
\end{abstract}

Keywords: Vulvar lesions, Haemangioma, Behcet's disease, Tuberculosis cutis orificialis

\section{INTRODUCTION}

Vulval symptoms are common and cause considerable distress for women. Symptoms are often chronic and can significantly affect the quality of women's lives including sexual function and wellbeing.

Anatomically it is a part of genitalia, composed of several folds including clitoral hood, labia majora and minora, hymen, and anal margin. ${ }^{1}$

Despite the frequency of vulval symptoms, women often find it difficult to seek medical advice. Research has shown that there are many reasons as to why women do not ask for help including:
That for cultural or emotional reasons as this part of the body is not much debated or discussed about in Indian women. That this region is not easily self-observable. The feeling that it is an embarrassing, uncomfortable or private matter. The belief that it is a normal part of getting older. Not being aware that there are treatments available. Not knowing how to initiate a conversation about these issues. Lack of knowledge that that signs can be observed elsewhere on the body, such as in lichen sclerosus or psoriasis when the lesion could be in vulva. Lack of knowledge that many vulval lesions can be secondary to large variety of diseases, such as digestive, hematological, immunological, and endocrine disorders. 
Pathologically vulva, as a part of genitalia, can be involved by specific disorders such as multifocal HPV lesions of or vaginal infection Vulva can also exhibit specific dermatological diseases. ${ }^{2}$ In addition, vulva can also exhibit signs of a large variety of diseases, such as digestive, hematological, immunological, and endocrine disorders. ${ }^{3}$ Hence any vulvar disorder could be a presentation of not only dermatological but also non dermatological condition.

\section{Aim}

To diagnose and treat abnormal vulvar lesions in women who attended Gynaec OPD of Dhiraj general hospital.

\section{Objectives}

- Identify whether presenting vulval lesion is dermatological or non-dermatological and to control the symptoms.

- Identify nature of the lesion (Whether it is inflammatory or neoplastic).

- Identify those Skin lesions not responding to treatment which require biopsy and further management.

\section{CASE REPORT}

It was a clinical descriptive study. Cases included women with vulvar lesions who attended Gynaec OPD at Dhiraj General Hospital affiliated to S.B.K.S. Medical Institute and Research Centre of Vadodara, Gujarat, India.

After taking their informed consent, all the women with vulvar lesions were thoroughly investigated to rule out any systemic illness causing vulvar lesion following biopsy was taken if solid growth or a non-healing lesion was present. Treatment was done according to the lesions.

\section{Inclusion criteria}

- All females who had symptoms like vaginal discharge, itching or discomfort associated a with vulvar lesion

- All females with visible lesion over vulva.

- All females with visible growth over vulva.

\section{Exclusion criteria}

- Women who had vaginitis or cervicitis.

- Women with diagnosed or recurrent vulval cancer.

- Women with asymptomatic or nonvisible lesions.

\section{Methodology}

The importance of history taking cannot be emphasized enough. The following points were considered while eliciting the history.
Whether symptoms were sudden or gradual in onset was asked.

Complaints of pain, discomfort, irritation, itching were commonly encountered.

Complaints of dyspareunia, discharge and whether the partner had any similar complaints were enquired about.

Since we dealt mostly with women from rural backgrounds who are not very good at accurate localisation of that part of their anatomy, whether the lesion was vulval or vaginal was ascertained.

Some women who had made close inspection with a hand mirror could describe the lesion whilst others did not contemplate looking down there.

Complaints of dysuria and increased urinary frequency too were looked out for as even in absence of urinary tract infection, it is painful to pass urine through an inflamed area.

Questions about any other concurrent problems such as psoriasis, chickenpox, and history of trauma or recent use of antibiotics were asked.

Also, whether or not the problem was recurrent was kept in mind.

Details of any personal or family history of atopic conditions (hay fever, asthma, eczema) were elicited. History of weight loss or prolonged cough helped us to conclude a tuberculous affection. History of diabetes was asked for in cases of recurrent vulvitis. Also, that Crohn's disease might as well have vulvar cutaneous involvement was kept in mind.

A thorough examination with adequate lighting was done, as changes can be subtle. Pelvic floor muscle tone was assessed if penetration was painful.

Examination of the rest of the body, including the mouth, for signs of lichen planus and the scalp, elbows, knees and nails for psoriasis was done .Eczema may be seen at any site. The conjunctiva was examined as it's affected in cases of Behcet's disease. Thorough examination of the lymph nodes was done keeping in mind possibility of TB or malignancy. Lower eyelids, malar area, upper lips were examined as they may be involved in syringomas.

When confronted with a vulvar lesion, it is helpful to "categorize" the lesion using a systematic pathway which decreases the chance of missing a diagnosis. Dermatological and neoplastic classification is beyond the scope of this paper and only unusual cases have been the focus. Thus the colour and presentation of the lesion was used to broadly classify the lesion and to reach a provisional diagnosis. 


\section{Visible white lesions}

- Lichen sclerosus

- Squamous cell hyperplasia

- Leukoplakia

- HPV

- VIN

- Squamous cell carcinoma of the vulva

\section{Red or dark lesions}

- Seborrheid dermatitis

- Psoriasis

- Lichen planus

- Lentigo

- Paget's disease

- Nevi

- VIN or cancer

- Malignant melanoma

When the diagnosis was not readily apparent by unaided (i.e., naked-eye) or colposcopic examination of the vulva, vulvar biopsies were performed for acetowhite changes and visible vulvar lesions such as ulceration or hyperpigmentation.

Mostly the diagnosis was done by inspection and clinical presentation. It was remembered that Patch testing may be necessary in cases of vulvar dermatitis.

\section{Serology}

CRP and ESR were done in patients who appeared to have tuberculous affection of the vulva.

$\mathrm{CBC}$ was routinely done in all cases.

ANA and HLA-B51 was done where if Behcet's disease was a possibility.

RPR (Rapid Plasma Reagin) was done in solitary, raised, firm, red papules with regional non-tender lymphadenopathy.

HIV testing was done in all cases routinely.

\section{Urine}

\section{PCR for Chlamydia (First pass urine test)}

PCR for Gonorrhoea (First pass urine test)

For non-healing lesions the patient was referred for biopsy of lesion. The mainstay of diagnosis is vulvar biopsy. Furthermore, all patients with a non-neoplastic vulvar epithelial disorder were checked at regular intervals. Areas of ulceration or foci of granulation or nodularity that developed were biopsied to exclude malignant change. The formation of hyperkeratotic plaques or erosions that do not respond to treatment aroused suspicions of malignancy.

\section{Case presentations}

Case 1

A 23 year old female, nulligravida came with complaints of growth on medial region of the right vulva for 6 years, when she had first noticed it as a reddish subcutaneous nodule. She ignored it despite its gradual clinical enlargement. There was no significant past or family history.

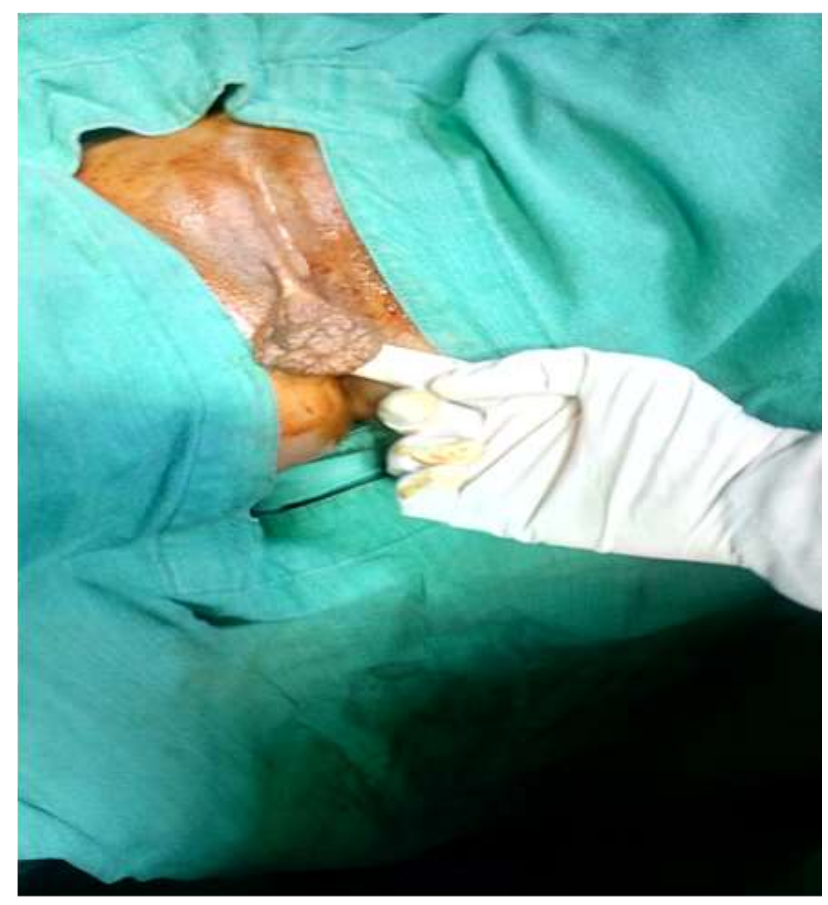

Figure 1: Haemangioma vulva.

Local examination revealed a pedunculated growth of 6 $\mathrm{cm} \times 3 \mathrm{~cm}$, non-tender with irregular margins. It was soft in consistency. There was no lymphadenopathy.

Trans-illumination test was positive.

Differential diagnosis was labial cyst, and mesenchymal tumors such as lipoma and liposarcoma.

USG showed ill-defined hypoechoic mass of heterogeneous echo texture with multiple cystic spaces within.

Colour Doppler was done which showed no feeder vessel from large vessel and slow venous flow.

Treatment: Simple tumor excision was done at the level of the stalk.

Histopathology report was suggestive of a haemangioma. 


\section{Case 2}

A 65 year old Para 4 Live 4 female presented with complaints of dysuria and ulceration over external genitalia for 1 year. It had grown slightly in size over the past year. She had no significant past, personal or family history. The patient had initially been given antibiotics elsewhere classifying it as an inflammatory lesion.

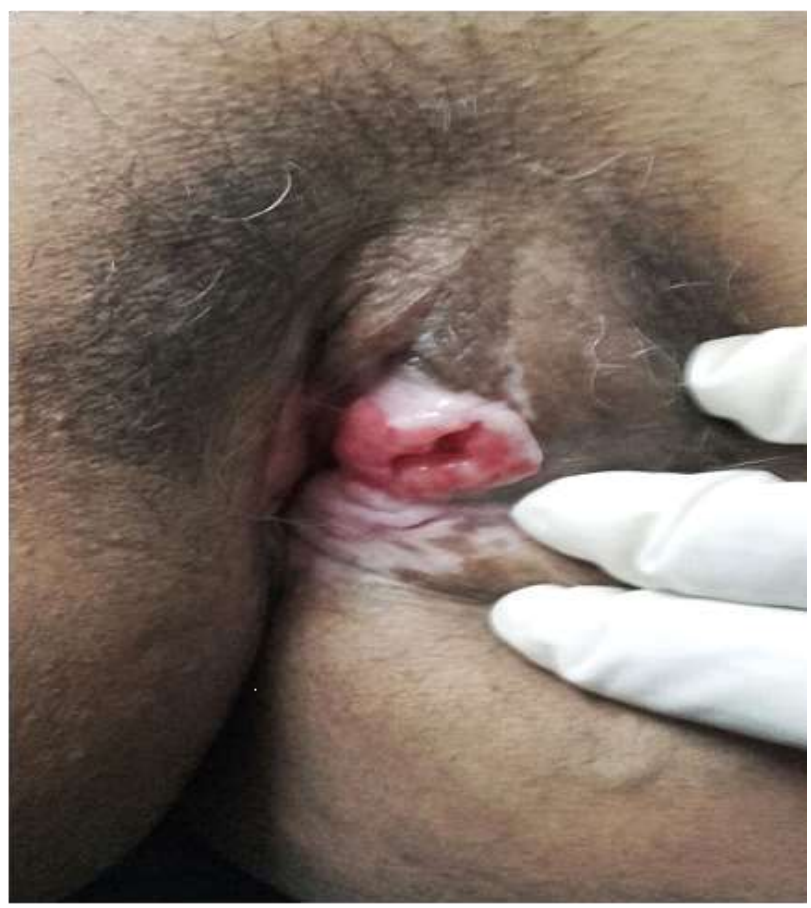

Figure 2: Squamous cell carcinoma.

Local examination revealed a growth with an ulcerated area of $4 \times 3 \mathrm{~cm}$ which involved the left labia minora, labia majora and clitoris. It was irregular, non-tender, and soft with erosions over the surface. Inguinal lymphadenopathy was palpable

Differential diagnosis: Squamous cell carcinoma, Basal cell carcinoma, melanoma, adenocarcinoma

Old age with a non-healing ulcer with non-tender lymphadenopathy led us to suspect that it could be malignant and biopsy was done. Biopsy report suggested well differentiated squamous cell carcinoma of the vulva and nodes were evaluated by FNAC. Staging work-up, including chest x-rays, and abdominal CT scan, did not show any sign of distant sites of disease.

Radical vulvectomy with bilateral inguinal lymphadenectomy with vulvar reconstruction was done.

The patient was referred to a higher centre for further treatment.
Case 3

A 25 years old G2P1L1 at 33 weeks of gestation with fever and rashes over left foot, hand, genital $\mathrm{n}$ oral lesions that developed over a month. There were episodes of similar ulcerations last year.

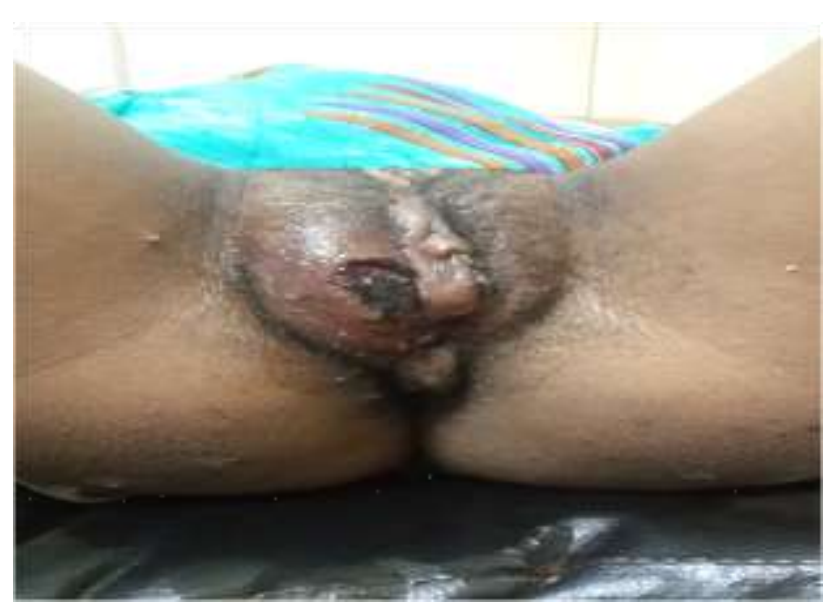

Figure 3: Behcet's disease afflicting vulva.

On clinical intraoral exam, multiple ulcers were observed, measuring approximately $0.5-1.2 \mathrm{~cm}$ in diameter, with the presence of an erythematous halo.

On examination of vulva multiple bluish red ulcers necrotic ulcers which were painful during palpation were seen, which had irregular margins were present over right labia, clitoris and fourchette. The labia were swollen and pushed the left labia to one side, rendering a distorted appearance.

Bilateral cervical, axillary and inguinal non tender lymphadenopathy was present.

Differential diagnosis: Malignancy, HIV/AIDS, magic Syndrome (mouth and Genital ulcers with inflamed cartilages), sarcoidosis, PFAPA syndrome (Periodic fever, aphthous stomatitis, pharyngitis and adenitis syndrome)

Histopathology from skin biopsy showed acute on chronic inflammation with necrotic areas and areas of leucocytoclastic vasculitis and was consistent with findings of Behcet's disease.

Management: Erythromycin (500mg) QID and clotrimazole mouth paint was given. Fluticasone ointment for local application was given.

Patient went into spontaneous labour at 36 weeks and delivered a female child of $1.9 \mathrm{kgs}$ her post-partum period was uneventful and the rashes healed over the next 2 weeks. 


\section{Case 4}

A 33 year old female P2, L2 came with complaints of a swelling over the vulva which was associated with mild pain. This swelling had grown in size over 1 year.

Local Examination showed a cyst of $1 \times 2 \mathrm{cms}$. Over the right labia minora. It was soft, cystic, and non-tender. No lymphadenopathy was present.

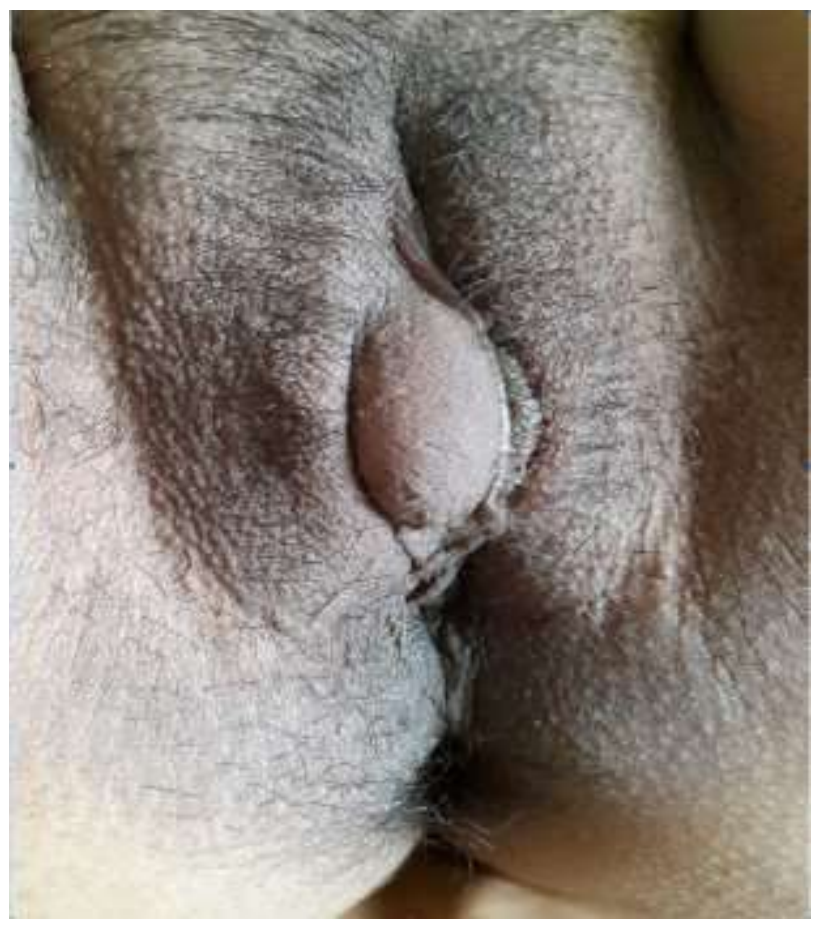

Figure 4: Bartholin's cyst.

Differential Diagnosis: Inclusion cyst, sebaceous cyst, Bartholin's cyst.

Diagnosis of Bartholin's cyst made by clinical evaluation and physical evaluation.

Marsupialisation was done and antibiotics were given.

\section{Case 5}

A 22 year old female patient presented with chronic painful ulcers of over genitalia and weight loss since 12 months. There was no history of persistent cough, though there were episodes of mild evening rise in temperature.

On examination: There were multiple painful ulcers involving the labia majora, minora and fourchette which were associated with seropurulent discharge. Multiple, enlarged, non-tender, mobile inguinal lymph nodes could be palpated.

Differential diagnosis: Tuberculous cutis orificialis, syphilis, deep fungal infections

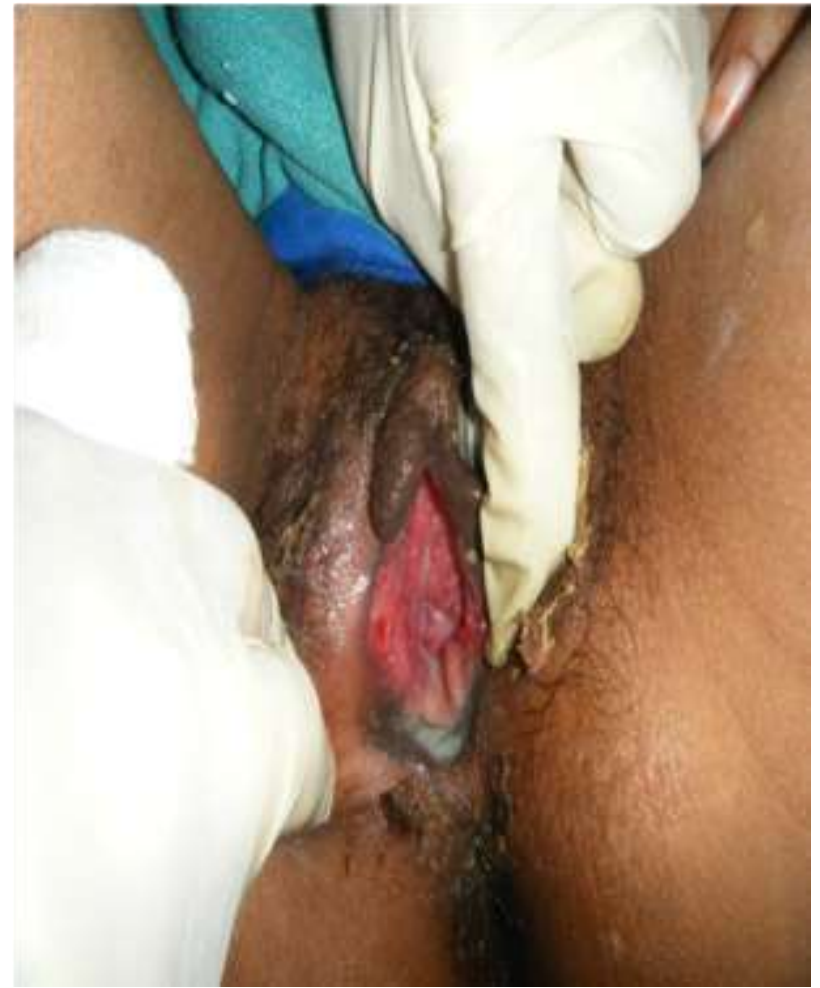

Figure 5A: Tuberculous cutis orificialis.

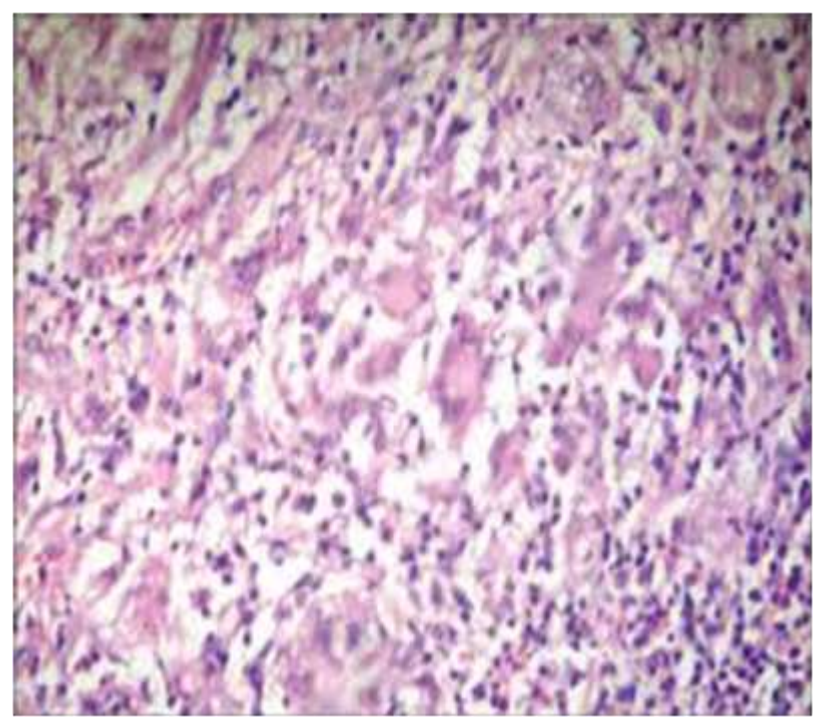

Figure 5B: Tuberculoid granulomas.

Mantoux test was positive. Erythrocyte sedimentation rate $(80 \mathrm{~mm} / \mathrm{h}$; normal $1-20)$ and low haemoglobin $(\mathrm{Hb}$ $10 \mathrm{~g} / \mathrm{dl}$; normal 12-16). Liver function, renal function and blood sugar were normal. Serology for sex transmitted diseases including syphilis, human immunodeficiency virus, hepatitis $\mathrm{B}, \mathrm{C}$ was negative.

Histopathology revealed tuberculoid granulomas with necrosis in the deeper dermis.

Acid-fast bacilli were seen in the granuloma and necrotic area by Ziehl-Neelsen stain. 


\section{Diagnosis - Tuberculous cutis orificialis}

The patient was started on standard anti-TB therapy, consisting of isoniazid $300 \mathrm{mg}$, rifampicin $600 \mathrm{mg}$, pyrazinamide $1000 \mathrm{mg}$, and sethambutol $900 \mathrm{mg}$ daily for 2 months, followed by an additional 4 months therapy with isoniazid and rifampicin.

\section{Case 6}

A 45 year old female patient presented with large verrucous plaque over suprapubic region extending to right labia majora for past 5 years. H/O occasional fluid discharge from lesions.

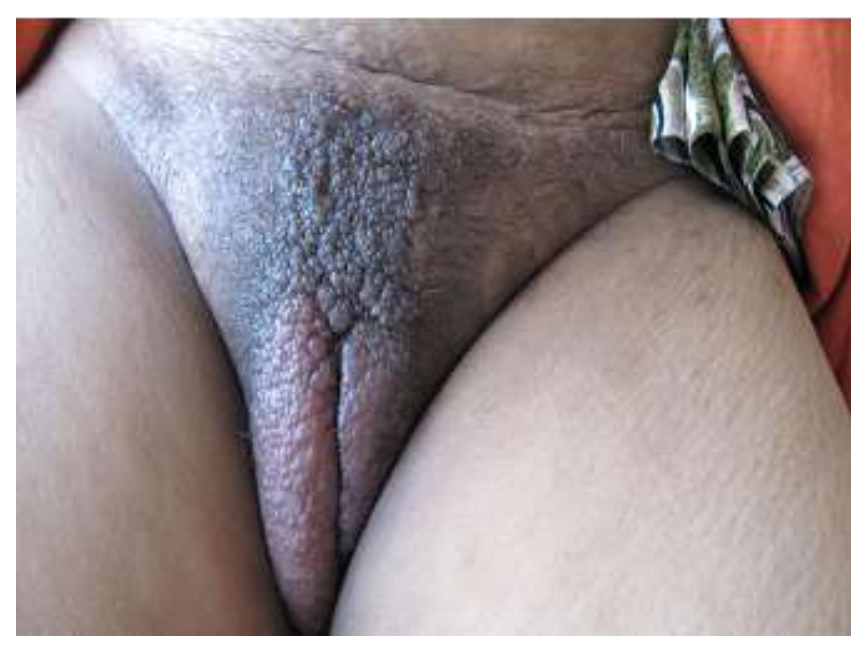

Figure 6A: Lymphedema vulva.

On examination indurated non-tender bilateral inguinal lymph nodes present.

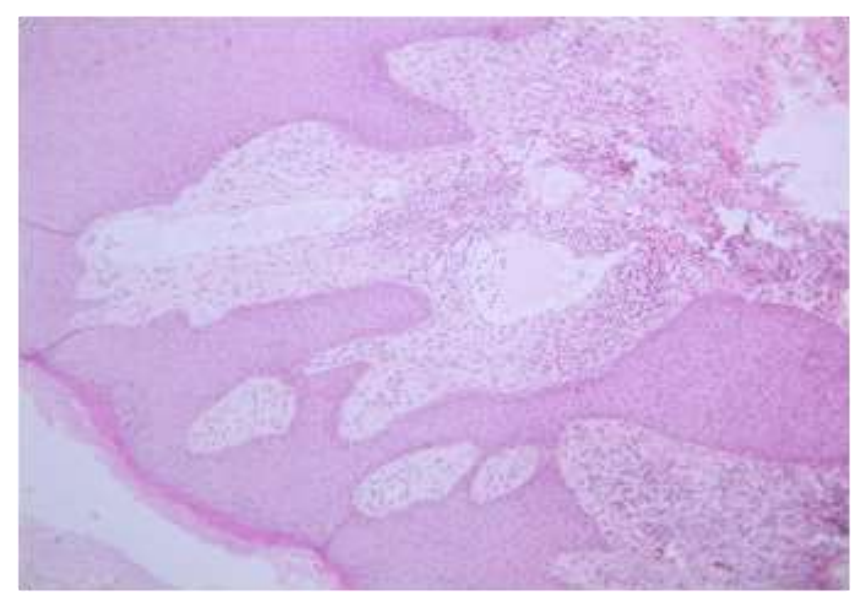

Figure 6B: Multiple dilated cystic spaces in the papillary dermis.

FNAC revealed features of tuberculous lymphadenitis.

Histopathology from the lesion revealed multiple dilated cystic spaces in the papillary dermis.
Diagnosis - Lymphedema of vulva secondary to tuberculosis.

\section{DISCUSSION}

The vulva is the part of the female genital tract located between the genitocrural folds laterally, the mons pubis anteriorly, and the anus posteriorly. This hollow structure contains the labia majora, labia minora, clitoris, vestibule, urinary meatus, vaginal orifice, hymen, Bartholin glands, and skene ducts. Microscopically, vulva is covered by different types of epithelia, depending on the area of interest, including, from its lateral to medial region, keratinized hair bearing skin, partially keratinized hairless skin, and, beyond Hart's line, mucous membrane of the vestibule. A large number and variety of adnexal structures are associated with vulvar skin in its different sections, such as pilosebaceous units, sebaceous and sweat glands, mucous secretory glands, muscle fibres, and deeper major or minor vestibular glands. Vicinity of underlying vascular structures can also modify vulvar aspects. Therefore, any component of blood and lymphatic vessels can be affected through malformations, tumors, or dystrophic changes. The epithelium of the vestibule is neither pigmented nor keratinized and contains eccrine gland. Considering anatomy and patient's own point of view, this region is of course not easily self-observable. ${ }^{2}$

From the above cases we can understand that the women coming with vulvar lesions to the OPD are few. To come to a reasonable diagnosis, it is very important that we follow a protocol or an outline, based on color, symptoms, concurrent lesions elsewhere and assessment of lymph nodes.

The history may be difficult to elicit because of anxiety about the diagnosis, frustration about ineffective treatment, secondary sexual dysfunction or resultant and often under-recognised depression.

When child presents with a condition that is usually sexually transmitted (such as genital warts or HSV), sexual activity needs to be considered, but it is not the only cause. Evidence of trauma, especially with a spurious explanation, is also suggestive. Other features may include behavioural disorders and inappropriate sexual behaviour.

Infectious disorders include diseases caused by known transmissible agents, such as viruses, bacteria, fungi, and protozoa. Non neoplastic epithelial disorders like Acanthosis nigricans, Behcet's disease, Contact dermatitis, Lichen sclerosis, Paget's disease, atopic dermatitis can also affect vulva.

Cystic lesions of vulva include bartholin gland cyst, skene duct cyst, and epithelial inclusion cyst. Other than cancer vulva, a solid non-neoplastic lesion includes fibroma, haemangioma, lipoma etc. 
Too often, women suffering from vulvar complains, especially itching, are still nowadays offered symptomatic treatment without even examination or diagnostic process, such as over-the-counter delivery of any cream. This may, in some cases, lead, even in young people, to delayed diagnosis of life-threatening diseases, such as squamous cell carcinoma (SCC). ${ }^{2}$

No screening strategies have been developed for the prevention of vulvar cancer through early detection of VIN.

Diagnosis is limited to visual assessment. Biopsy is indicated for most pigmented and non-healing vulvar lesions. ${ }^{5}$ Vulval cancer is an aggressive cancer with poor prognosis and few treatment options hence by thoroughly diagnosing and treating these premalignant and benign lesions we can prevent cancer.

\section{CONCLUSION}

Vulva can also exhibit signs of a large variety of diseases, such as digestive, hematological, immunological, and endocrine disorders. Hence consider any vulvar disorder as a potential expression of a very large panel of diseases.

In the presence of vulvar complains, a diagnostic pathway including systematic physical examination is always mandatory to detect SCC early.

Important to note localization, boarder asymmetry, number, color and distribution of abnormal lesion on examination.
A biopsy always indicated in a non-healing ulcerative or hypertrophied lesion not responding to medical treatment.

Women should be educated to avoid ODC drugs or creams when they face vulval lesions.

Funding: No funding sources

Conflict of interest: None declared

Ethical approval: Not required

\section{REFERENCES}

1. Fistarol SK, Itin PH. Diagnosis and treatment of lichen sclerosus. American Journal of Clinical Dermatology. 2013;14(1):27-47.

2. Doyen J. Vulvar Skin Disorders throughout Lifetime: About Some Representative Dermatoses. BioMed Research International. 2014; Article ID 595286.

3. Marzano AV, Borghi A, Stadnicki A, Crosti C, Cugno M. Cutaneous manifestations in patients with inflammatory bowel diseases: pathophysiology, clinical features, and therapy. Inflamm Bowel Dis. 2014;20(1):213-27.

4. Hill DA. Vulvar Diseases in Primary Care, The Visible Lesion: Is it Cancer? Retrieved from http://www.healthieryou.com/c-vul.html.

5. Management of Vulvar intraepithelial neoplasia. Committee Opinion No. 509. American College of Obstetricians and Gynecologists. Obstet Gynecol. 2011;118:1192-4.

Cite this article as: Kothari $\mathrm{A}$, Parekh $\mathrm{U}$, Mahajan R. Abnormal vulval lesions: diagnosis and management. Int J Reprod Contracept Obstet Gynecol 2016;5:xxx-Xx. 